\title{
Ro-Translational Cavity Cooling of Dielectric Rods and Disks
}

\author{
Benjamin A. Stickler, ${ }^{1}$ Stefan Nimmrichter, ${ }^{1,2}$ Lukas Martinetz, ${ }^{1}$ \\ Stefan Kuhn, ${ }^{3}$ Markus Arndt, ${ }^{3}$ and Klaus Hornberger ${ }^{1}$ \\ ${ }^{1}$ Faculty of Physics, University of Duisburg-Essen, Lotharstraße 1, 47048 Duisburg, Germany \\ ${ }^{2}$ Centre for Quantum Technologies, National University of Singapore, Singapore \\ ${ }^{3}$ Faculty of Physics, University of Vienna, VCQ, Boltzmanngasse 5, 1090 Vienna, Austria
}

\begin{abstract}
We study the interaction of dielectric rods and disks with the laser field of a high finesse cavity. The quantum master equation for the coupled particle-cavity dynamics, including Rayleigh scattering, is derived for particle sizes comparable to the laser wavelength. We demonstrate that such anisotropic nanoparticles can be captured from free flight at velocities higher than those required to capture dielectric spheres of the same volume, and that efficient ro-translational cavity cooling into the deep quantum regime is achievable.
\end{abstract}

\section{INTRODUCTION}

Laser cooling and controlling the ro-translational degrees of freedom of a nanoparticle in vacuum is a challenging task [1] with far-reaching implications: Levitated dielectrics in a high-finesse cavity can be used as ultrasensitive force sensors with atto- or even zeptonewton sensitivity [2 [5]. In addition, preparing nanoscale dielectrics in the deep quantum regime may allow to address fundamental questions, such as the thermalization of a single particle [6 9] or the validity of the quantum superposition principle at high mass scales [10, 11.

In the case of small molecules with a sharp internal transition, the ro-translational motion can be laser cooled by exploiting the methods developed for atoms [12 14]. Micron-sized particles in solution and low vacuum can be trapped and manipulated rotationally with optical tweezers and vortex beams [15]23. A first step towards controlling the ro-translational state of a nanometer-sized rod in high vacuum was demonstrated only recently [24].

Here, we show that nanoscale rods and disks are excellent candidates for cavity cooling [25 27, possibly even into their ro-translational ground state. This is due to a number of fortuitous properties: (i) The anisotropic shape of a dielectric appreciably enhances the effective interaction with the cavity as compared to a sphere of the same volume, and (ii) for sufficiently red-detuned cavities, efficient cooling takes place for all orientations and positions of the particle. In addition, (iii) their rotranslational motion can be tracked from the scattered light, since the particle's position and orientation is encoded in the polarization and intensity, and $(i v)$ the final temperature is well below the ro-translational level spacing in the trap potential.

The finite extension of the dielectric must be taken into account when describing the interaction between a nanoparticle and the field of a high finesse cavity since the laser intensity varies on the length-scale of the particles 24, 28 30. Rather than using numerical techniques [31, 32] or iterative methods [33], we exploit that the considered particles are thin, allowing us to analytically derive the internal polarization field [34] and thus the optical potential. Here, it is crucial that we adopt the direction of the internal polarization field from the exact electrostatic solution [35] and therefore correctly account for the anisotropic susceptibility of the particle. Our resulting analytic expressions for the Markovian particlecavity dynamics and the scattered light intensity provide the theoretical toolbox required to carry out cavity experiments with dielectric nanorods and nanodisks in high vacuum.

We start by deriving the optical potential of a dielectric rod or disk in a standing wave cavity mode, which enters the Markovian master equation for the combined state of particle and field mode. The cooling rate due to the retarded back-action of the light field is then extracted from this equation. It also determines the threshold velocity for capturing a particle in free flight. We account for recoil heating of the particle by light scattering into the vacuum modes by deriving the orientation-dependent Rayleigh scattering operators, and determine the final temperature of a deeply trapped particle.

\section{CAVITY INDUCED POTENTIAL}

We consider a thin dielectric rod or disk, modeled as a cylinder of length $\ell$, radius $a$, and mass $M$ with moment of inertia perpendicular to the symmetry axis, $I_{\mathrm{r}}=M \ell^{2} / 12$ or $I_{\mathrm{d}}=M a^{2} / 4$, respectively. The particle propagates through the field of a standing wave Gaussian cavity mode of waist $w_{0}$ and wavelength $2 \pi / k$, see Fig. 1. The cavity mode is driven by a pump laser of angular frequency $\omega_{\mathrm{p}}$ and power $P_{\mathrm{p}}=\hbar \omega_{\mathrm{p}} \eta^{2} / 2 \kappa$ where $\eta$ is the pump rate [36]. Choosing the $z$-axis along the cavity axis and the $x$-axis in direction of the cavity mode polarization, the local cavity field at position $\mathbf{r}^{\prime}=\left(x^{\prime}, y^{\prime}, z^{\prime}\right)$ reads $\mathbf{E}_{\text {in }}=\sqrt{2 \hbar \omega_{\mathrm{p}} / \varepsilon_{0} V_{\mathrm{c}}} b f\left(\mathbf{r}^{\prime}\right) \cos \left(k z^{\prime}\right) \mathbf{e}_{x}$ where $f\left(\mathbf{r}^{\prime}\right)$ is the Gaussian envelope with waist $w_{0}$ and $V_{\mathrm{c}}$ and $b$ denote the mode volume and the dimensionless field amplitude, respectively.

Evaluating the optical force and torque exerted by the laser field on the dielectric requires knowledge of the macroscopic polarization field $\mathbf{P}$ inside the particle [37, 38. In general, the internal field must be determined numerically if the particle's extension is compara- 


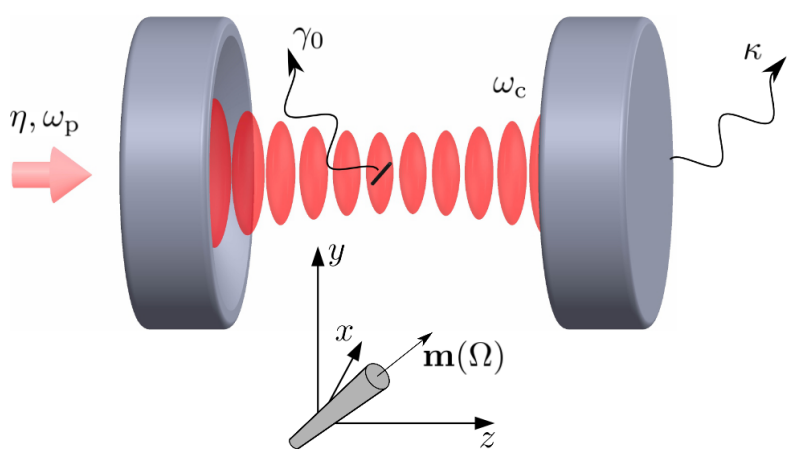

Figure 1. (Color online) A thin dielectric rod or disks traverses the laser field of a high finesse cavity with resonance frequency $\omega_{\mathrm{c}}$, driven with pump rate $\eta$ and pump frequency $\omega_{\mathrm{p}}$. The cavity detuning is $\Delta=\omega_{\mathrm{p}}-\omega_{\mathrm{c}}$ and $\kappa$ and $\gamma_{0}$ are the cavity linewidth and the Rayleigh scattering rate, respectively. The orientation vector of the dielectric's symmetry axis is denoted by $\mathbf{m}(\Omega)$.

ble to the laser wavelength 32. However, in the present case one can exploit that the particle is very thin in at least one direction in order to derive an approximate analytic expression for the polarization field (generalized Rayleigh-Gans approximation) 34. Specifically, this is possible for rods and disks of (real) dielectric permittivity $\varepsilon_{\mathrm{r}}$ since $\pi k^{2} a^{2}\left(\varepsilon_{\mathrm{r}}-1\right) \ll 1$ or $k \ell\left(\varepsilon_{\mathrm{r}}-1\right) \ll 1$, respectively.

The resulting internal polarization field acquires the position dependence of the external field but it is rotated according to the dielectric's susceptibility tensor. The internal field is the exact solution to Maxwell's equations in the limit of infinitesimally thin particles and it provides a good approximation for the present scenario [24]. In particular, the field accounts correctly for the anisotropic susceptibility of the nanoparticles because its polarization is obtained by solving the corresponding electrostatic problem 34. In the case of rods, the components of the susceptibility tensor perpendicular and orthogonal to the symmetry axis are $\chi_{\|}^{\mathrm{r}}=\varepsilon_{\mathrm{r}}-1$ and $\chi_{\perp}^{\mathrm{r}}=2\left(\varepsilon_{\mathrm{r}}-1\right) /\left(\varepsilon_{\mathrm{r}}+1\right)$ while $\chi_{\|}^{\mathrm{d}}=\left(\varepsilon_{\mathrm{r}}-1\right) / \varepsilon_{\mathrm{r}}$ and $\chi_{\perp}^{\mathrm{d}}=\varepsilon_{\mathrm{r}}-1$ for disks [35]. We denote the anisotropy by $\Delta \chi=\chi_{\|}-\chi_{\perp}$ and the maximal value by $\chi_{\mathrm{m}}=\varepsilon_{\mathrm{r}}-1$. Note that the orientationally averaged susceptibility of a dielectric rod or disk thus exceeds the susceptibility of a dielectric sphere. With the above notation, the polarization field is proportional to $\chi_{\perp} \mathbf{e}_{x}+\Delta \chi\left(\mathbf{e}_{x} \cdot \mathbf{m}\right) \mathbf{m}$ where $\mathbf{m}$ is the direction of the dielectric's symmetry axis. This dependence on the dielectric's orientation is familiar from anisotropically polarizable point-like particles [39].

The particle-cavity interaction potential can now be calculated by integrating the potential energy density derived from cavity perturbation theory [30, 40, 41] averaged over one optical cycle, $-\mathbf{P} \cdot \mathbf{E}_{\text {in }}^{*} / 4$, or, equivalently, from the averaged force- and torque densities [38. Denoting the center-of-mass position of the dielectric by $\mathbf{r}$ and its orientation by $\mathbf{m}(\Omega)$, with $\Omega=(\alpha, \beta, \gamma)$ the Eu- ler angles in the $z-y^{\prime}-z^{\prime \prime}$ convention, we find the optical potential as

$$
\begin{aligned}
V(\mathbf{r}, \Omega)= & \hbar U_{0}|b|^{2} f^{2}(\mathbf{r})\left[\frac{\chi_{\perp}}{\chi_{\mathrm{m}}}+\frac{\Delta \chi}{\chi_{\mathrm{m}}}\left(\mathbf{m}(\Omega) \cdot \mathbf{e}_{x}\right)^{2}\right] \\
& \times\left[\frac{1}{2}+\frac{1}{2} \cos (2 k z) S\left(\mathbf{m}(\Omega), \mathbf{e}_{z}\right)\right] .
\end{aligned}
$$

Here, $U_{0}=-\omega_{\mathrm{p}} \chi_{\mathrm{m}} V_{0} / 2 V_{\mathrm{c}}$ denotes the coupling frequency with $V_{0}$ the dielectric's volume. The orientationdependent shape function $S(\mathbf{m}, \mathbf{n})$ accounts for the particle's finite extension and reads for rods and disks, respectively, as

$$
\begin{aligned}
S_{\mathrm{r}}(\mathbf{m}, \mathbf{n}) & =\frac{\sin (k \ell \mathbf{m} \cdot \mathbf{n})}{k \ell \mathbf{m} \cdot \mathbf{n}}, \\
S_{\mathrm{d}}(\mathbf{m}, \mathbf{n}) & =\frac{J_{1}(2 k a|\mathbf{m} \times \mathbf{n}|)}{k a|\mathbf{m} \times \mathbf{n}|},
\end{aligned}
$$

where $J_{1}(\cdot)$ denotes a Bessel function of the first kind. Both functions (2) take on their maximum value if their arguments vanish and, thus, it can be seen from (1) that rods tend to align with the field polarization, $\mathbf{m}=\mathbf{e}_{x}$, while disks align with the cavity axis, $\mathbf{m}=\mathbf{e}_{z}$. In the limit of small dielectrics, $k \ell \ll 1$ or $k a \ll 1$, the potential of an anisotropic point-like particle in a standing wave laser field [39] is recovered and the potential (1) becomes proportional to the local laser intensity.

\section{MASTER EQUATION FOR DIELECTRIC AND CAVITY}

A dielectric particle moving through the cavity modifies the laser intensity by effectively shifting the cavity resonance frequency $\omega_{\mathrm{c}}$ as well as enhancing the cavity loss rate. While this retarded reaction of the cavity on the dielectric's motion can cool the particle's motional state, Rayleigh scattering of cavity photons off the dielectric leads to recoil heating. Compared to other decoherence mechanisms [41, recoil heating dominates for deeply trapped particles and thus determines the steady state temperature. The coupled particle-cavity dynamics can be described with the help of a Markovian quantum master equation for the total state operator $\rho$. This equation can be derived by coupling the particle-cavity system to the infinite bath of empty vacuum modes, which are then traced out in the Born-Markov approximation [42. Here, scattering enhanced coupling between vacuum modes [30] can be safely neglected since we consider very thin particles, for which the scattering rate is sufficiently low. Denoting by $\hat{\mathbf{r}}$ and $\hat{\Omega}$ the ro-translational coordinate operators and by $b$ the cavity field operator, one obtains

$$
\begin{aligned}
\partial_{t} \rho= & -\frac{i}{\hbar}\left[\mathrm{H}_{\mathrm{p}}, \rho\right]-i U_{0}\left[v(\hat{\mathbf{r}}, \hat{\Omega}) \mathbf{b}^{\dagger} \mathrm{b}, \rho\right]+\mathcal{L}_{\mathrm{c}} \rho \\
& +\gamma_{0} \sum_{s=1,2} \int_{S_{2}} \frac{\mathrm{d}^{2} \mathbf{n}}{4 \pi}\left[\mathrm{L}_{\mathbf{n} s} \rho \mathrm{L}_{\mathbf{n} s}^{\dagger}-\frac{1}{2}\left\{\mathrm{~L}_{\mathbf{n} s}^{\dagger} \mathrm{L}_{\mathbf{n} s}, \rho\right\}\right]
\end{aligned}
$$


where $\mathrm{H}_{\mathrm{p}}$ is the free particle Hamiltonian and $\mathcal{L}_{\mathrm{c}} \rho$ describes the unperturbed cavity dynamics,

$$
\mathcal{L}_{\mathrm{c}} \rho=i\left[\Delta \mathrm{b}^{\dagger} \mathrm{b}+i \eta\left(\mathrm{b}-\mathrm{b}^{\dagger}\right), \rho\right]+\kappa\left(2 \mathrm{~b} \rho \mathrm{b}^{\dagger}-\left\{\mathrm{b}^{\dagger} \mathrm{b}, \rho\right\}\right)
$$

with $v(\mathbf{r}, \Omega)=V(\mathbf{r}, \Omega) / \hbar U_{0}|b|^{2}$ the field-independent part of the interaction potential (1). The position- and orientation-dependent Lindblad operators of Rayleigh scattering into polarization direction $\boldsymbol{\epsilon}_{\mathbf{n} s}$ with scattering rate $\gamma_{0}=c \chi_{\mathrm{m}}^{2} V_{0}^{2} k^{4} / 6 \pi V_{\mathrm{c}}$, are $\mathrm{L}_{\mathbf{n} s}=\mathrm{b} A_{\mathbf{n} s}[\hat{\mathbf{r}}, \mathbf{m}(\hat{\Omega})]$ with

$$
A_{\mathbf{n} s}(\mathbf{r}, \mathbf{m})=\sqrt{\frac{3}{8}} f(\mathbf{r}) \boldsymbol{\epsilon}_{\mathbf{n} s} \cdot\left[\frac{\chi_{\perp}}{\chi_{\mathrm{m}}} \mathbf{e}_{x}+\frac{\Delta \chi}{\chi_{\mathrm{m}}}\left(\mathbf{m} \cdot \mathbf{e}_{x}\right) \mathbf{m}\right] e^{-i k \mathbf{n} \cdot \mathbf{r}}\left(e^{i k z} S\left[\mathbf{m},\left(\mathbf{e}_{z}-\mathbf{n}\right) / 2\right]+e^{-i k z} S\left[\mathbf{m},\left(\mathbf{e}_{z}+\mathbf{n}\right) / 2\right]\right)
$$

They are diagonal in the ro-translational degrees of freedom and thus tend to localize the state in position and orientation. While the operator (5) transfers a superposition of the momentum kicks $\hbar k\left(\mathbf{n} \pm \mathbf{e}_{z}\right)$ on the dielectric's center of mass, its action on the orientational degrees of freedom is more intricate because the angle operators $\hat{\Omega}$ are not the generators of angular momentum kicks but of the canonically conjugate momentum translations [43, 44]. Thus, an eigenstate $\left|m_{\alpha}, m_{\beta}, m_{\gamma}\right\rangle$ of the canonical momentum operator $\hat{p}_{\Omega}$ conjugate to the Euler angles $\Omega$ transforms under the action of (5) into a superposition of shifted eigenstates, each weighted with the corresponding Fourier coefficient of the Lindblad operator. Nevertheless, it will turn out that the Rayleigh scattering dissipator describes diffusive ro-translational motion for deeply trapped nanoparticles. In addition, in the limit of small, isotropically polarizable point particles [28, 41] the operators (5) turn proportional to the local standing wave profile.

\section{SCATTERED LIGHT INTENSITY}

The light scattered by the dielectric can be utilized to track its ro-translational motion [24]. Specifically, a detector at position $R \mathbf{n}$ measures the intensity

$$
I_{\mathbf{n}}(\mathbf{r}, \Omega)=\frac{\hbar \omega_{\mathrm{p}} \gamma_{0}|b|^{2}}{4 \pi R^{2}} \sum_{s=1,2}\left|A_{\mathbf{n} s}[\mathbf{r}, \mathbf{m}(\Omega)]\right|^{2}
$$

as derived from the electric field integral equation 34 in the far-field limit. The polarization of the scattered field depends only on the dielectric orientation since it is orthogonal to the direction of its internal polarization field.

\section{EQUATIONS OF MOTION}

In order to assess under which conditions cavity cooling and trapping of the dielectric is possible, we determine the local cooling rate from the classical equations of motion. Denoting by $\left(\mathbf{p}, p_{\Omega}\right)$ the canonically conjugate momentum coordinates, the classical equations of motion are obtained from the master equation (3) by replacing all operators by their expectation values,

$$
\begin{aligned}
\dot{b} & =i\left(\Delta-U_{0} v\right) b-\left(\kappa+\frac{\gamma_{\mathrm{sc}}}{2}\right) b+\eta \\
\dot{\Omega} & =\partial_{p_{\Omega}} H_{\mathrm{p}}, \quad \text { and } \quad \dot{\mathbf{r}}=\partial_{\mathbf{p}} H_{\mathrm{p}} \\
\dot{p}_{\Omega} & =-\partial_{\Omega} V+\hbar \gamma_{0}|b|^{2} \sum_{s=1,2} \int_{S_{2}} \frac{\mathrm{d}^{2} \mathbf{n}}{4 \pi} \operatorname{Im}\left(A_{\mathbf{n} s}^{*} \partial_{\Omega} A_{\mathbf{n} s}\right)
\end{aligned}
$$

and

$$
\dot{\mathbf{p}}=-\partial_{\mathbf{r}} V+\hbar \gamma_{0}|b|^{2} \sum_{s=1,2} \int_{S_{2}} \frac{\mathrm{d}^{2} \mathbf{n}}{4 \pi} \operatorname{Im}\left(A_{\mathbf{n} s}^{*} \partial_{\mathbf{r}} A_{\mathbf{n} s}\right)(7 \mathrm{c})
$$

where we introduced the total scattering rate as a function of position and orientation,

$$
\gamma_{\mathrm{sc}}(\mathbf{r}, \Omega)=\gamma_{0} \sum_{s=1,2} \int_{S_{2}} \frac{\mathrm{d}^{2} \mathbf{n}}{4 \pi}\left|A_{\mathbf{n s}}[\mathbf{r}, \mathbf{m}(\Omega)]\right|^{2}
$$

Equation 7a describes the retarded reaction of the light field on the particle dynamics. The equations of motion of the particle $(7 \mathrm{~b}$ ) and $(7 \mathrm{c})$ contain the conservative optical potential (1) as well as the non-conservative radiation pressure due to Rayleigh scattering (5). This contribution vanishes close to the minimum of the potential (1); it vanishes everywhere for isotropic point particles.

The particle-cavity equations (7) must be solved numerically in general. However, if the particle is not yet deeply trapped, we can neglect Rayleigh scattering. Assuming further that the particle moves sufficiently slowly, such that the cavity reacts nearly instantaneously, we expand the cavity amplitude to first order in all velocities and angular momenta. Thus, we obtain nonconservative equations describing the dissipative dynamics of the nanoparticle. In general, the different degrees of freedom are strongly coupled and exchange energy, such that it is not useful to define a friction rate for the individual coordinates. Nevertheless, by adapting Liouville's theorem one can calculate the rate at which an infinitesimal phase space volume centered at $(\mathbf{r}, \Omega)$ expands or 

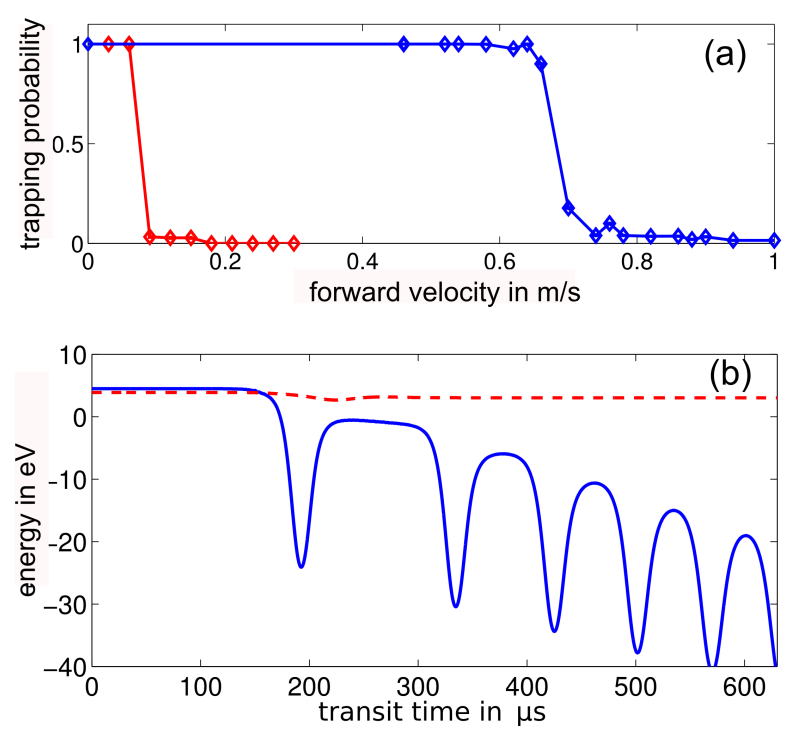

Figure 2. (Color online) (a) Trapping probability as function of the forward velocity for a silicon rod (blue; $\ell=800$ $\mathrm{nm}, a=25 \mathrm{~nm}$ ) and a sphere (red; $R \simeq 72 \mathrm{~nm}$ ) of the same volume. The nanoparticle is launched towards the cavity with forward velocity $v_{x}$. In order to obtain the trapping probability we solve the classical equations of motion (7) for several thousand initial conditions for each $v_{x}$. The transverse velocity $v_{z}$ is uniformly distributed within $5 \%$ of the forward velocity $v_{x}$ assuming a collimated beam. We neglect the weak $y$-dependence, setting $v_{y}=0$ and $y=0$, and assume the orientational degrees of freedom to be microcanonically distributed with a total rotation frequency of 1 $\mathrm{MHz}$. For the cavity, we chose the following realizable parameters: $\lambda=1.56 \mu \mathrm{m}, \kappa=0.78 \mathrm{MHz}, P_{\text {in }}=10 \mathrm{~mW}, \Delta=-1.2 \kappa$, $F=330000$. In (b) we show the total energy for a sample trajectory with $v_{x}=0.5 \mathrm{~m} / \mathrm{s}$ and $v_{z}=-0.3 \mathrm{~m} / \mathrm{s}$. The dielectric rod (blue) is captured and cooled while a sphere (red dashed) of the same volume traverses the cavity almost unaffected. Negative energies indicate that the particle is captured.

contracts [45],

$$
\begin{aligned}
\Gamma(\mathbf{r}, \Omega) & =\frac{4 \hbar \kappa \eta^{2} U_{0}^{2}\left[\Delta-U_{0} v(\mathbf{r}, \Omega)\right]}{\left(\kappa^{2}+\left[\Delta-U_{0} v(\mathbf{r}, \Omega)\right]^{2}\right)^{3}} \\
& \times\left(\frac{\left[\partial_{\mathbf{r}} v(\mathbf{r}, \Omega)\right]^{2}}{M}+\frac{\left[\partial_{\alpha} v(\mathbf{r}, \Omega)\right]^{2}}{I \sin ^{2} \beta}+\frac{\left[\partial_{\beta} v(\mathbf{r}, \Omega)\right]^{2}}{I}\right)(9)
\end{aligned}
$$

Notably, this rate is everywhere negative if the laser is sufficiently far red-detuned, i.e. for $\Delta<U_{0}$, and thus cooling occurs irrespective of the particle's position and orientation. This generalizes the results obtained for cavity cooling of point particles [46, 47]. The cooling rate (9) vanishes if the particle reaches the mechanical equilibrium, where the partial derivatives of $v(\mathbf{r}, \Omega)$ are zero. This could be circumvented by resorting to well established techniques such as two-mode [41, 48] or feedbackcooling 49 52.

The facts that cooling occurs almost everywhere for $\Delta<U_{0}$ and that the effective interaction strength is enhanced due to the anisotropy have a remarkable con- sequence: They facilitate trapping of rods and disks at velocities at which corresponding spheres would traverse the cavity almost unaffected. This is demonstrated in Fig. 2, which shows the trapping probability as a function of the forward velocity for dielectric rods and spheres of the same volume together with the total energy along one sample trajectory.

\section{COOLING LIMIT}

Recoil heating due to Rayleigh scattering prevents the dielectric from being cooled to its absolute ground state. To determine the steady-state temperature, we study the quantum master equation (3) in the case that the particle is already deeply trapped. For simplicity, we consider a moderately sized particle, $k \ell \simeq 1$ or $k a \simeq 1$, for which the interaction potential (1) can be approximated harmonically around its single minimum implying that the ro-translational degrees of freedom are only coupled via the cavity field. (If a linear coupling between the different degrees of freedom is required by a specific protocol this can be achieved by illumination with a second laser.)

For a dielectric rod aligned with the field polarization axis, $\mathbf{m}\left(\Omega_{0}\right)=\mathbf{e}_{x}$, at a position of maximum laser intensity, $\mathbf{r}_{0}=0$, the harmonic frequencies are

$$
\begin{aligned}
& \omega_{z}^{\mathrm{r}}=\sqrt{\frac{2 \hbar\left|U_{0}\right|\left|b_{0}\right|^{2} k^{2}}{M}}, \omega_{\alpha}^{\mathrm{r}}=\sqrt{\frac{2 \hbar\left|U_{0}\right|\left|b_{0}\right|^{2} \Delta \chi}{I \chi_{\|}}}, \\
& \omega_{\beta}^{\mathrm{r}}=\sqrt{\frac{2 \hbar\left|U_{0}\right|\left|b_{0}\right|^{2}}{I}\left(\frac{\Delta \chi}{\chi_{\|}}+\frac{(k \ell)^{2}}{12}\right)} .
\end{aligned}
$$

In a similar fashion, a deeply trapped disk has its surface aligned with the cavity axis, $\mathbf{m}\left(\Omega_{0}\right)=\mathbf{e}_{z}$, at a position of maximum laser intensity and the frequencies are $\omega_{z}^{\mathrm{d}}=\omega_{\beta}^{\mathrm{d}}=\omega_{z}^{\mathrm{r}}$ together with $\omega_{\alpha}^{\mathrm{d}}=0$. Here $b_{0}=\eta /\left(\kappa+\gamma_{\mathrm{sc}}^{0} / 2+i\left[\Delta-U_{0}\right]\right)$ denotes the steadystate cavity amplitude in the potential minimum with $\gamma_{\mathrm{sc}}^{0}=\gamma_{\mathrm{sc}}\left(\mathbf{r}_{0}, \Omega_{0}\right)$ the corresponding Rayleigh scattering rate. The frequencies in the transversal direction $(x, y)$ can be safely neglected because the laser waist $w_{0}$ is typically much larger than the wavelength. The frequencies (10) are of the same order of magnitude exceeding the cavity linewidth $\kappa$. Note that the trapping frequency $\omega_{z}$ is determined by the maximum susceptibility $\chi_{\mathrm{m}}=\varepsilon_{\mathrm{r}}-1$ rather than by the average value.

Similarly, we expand the Lindblad operators harmonically around the potential minimum. A straightforward calculation demonstrates that the remaining Lindblad operators are linear in the field operator $b$ as well as in the position operators $\hat{z}$ and $\hat{\Omega}$ and thus they describe diffusive motion. One can determine an approximate expression for the steady-state temperature from the resulting relations between steady-state operator expectation values. Defining temperature as the energy difference between the steady-state energy expectation value and the energy minimum divided by Boltzmann's constant, its 
steady-state value is given by the recoil limit

$$
T_{\nu}=\frac{\gamma_{0} \hbar^{2}\left|b_{0}\right|^{2}}{2 M_{\nu}\left(\kappa+\gamma_{\mathrm{sc}}^{0} / 2\right) k_{\mathrm{B}}} \sum_{s=1,2} \int_{S_{2}} \frac{\mathrm{d}^{2} \mathbf{n}}{4 \pi}\left|\partial_{\nu} A_{\mathbf{n} s}^{0}\right|^{2}
$$

for $\nu=z, \alpha, \beta$ with $M_{z}=M, M_{\alpha, \beta}=I$, and $\partial_{\nu} A_{\mathbf{n}}^{0}$ the derivative in direction $\nu$ of $A_{\mathbf{n}}$ evaluated at the potential minimum. In particular, for small particles, $k \ell \ll 1$, one has $T_{z} \simeq \gamma_{0} \hbar^{2} k^{2}\left|b_{0}\right|^{2} / 5 M \kappa_{\text {eff }} k_{\mathrm{B}}$ and $T_{\alpha, \beta} \simeq$ $\gamma_{0} \hbar^{2} \Delta \chi^{2}\left|b_{0}\right|^{2} / 2 I \chi_{\mathrm{m}}^{2} \kappa_{\text {eff }} k_{\mathrm{B}}$ where $\kappa_{\text {eff }}=\kappa+\gamma_{0} / 2$.

As an example we consider the silicon rods from Fig. 2. They are strongly coupled to the cavity, $\left|U_{0}\right| / \kappa \simeq 1.1$, yielding the final temperature $T_{z} \simeq 14 \mu \mathrm{K}$, which corresponds to a mean occupation number in the harmonic potential of $n_{z} \simeq 0.16$. In a similar fashion, we obtain for the rotational degrees of freedom $T_{\alpha} \simeq 31 \mu \mathrm{K}$ $\left(n_{\alpha} \simeq 0.34\right)$ and $T_{\beta} \simeq 29 \mu \mathrm{K}\left(n_{\beta} \simeq 0.23\right)$. This demonstrates that reaching the ro-translational ground state should indeed be possible.

\section{CONCLUSIONS}

Our findings open the door for numerous experiments and applications: The control gained over center of mass and the orientational degrees of freedom can be used for inertial sensing [2]. By monitoring the scattered light in- tensity one can track the dynamical impact induced by a background gas, allowing the direct observation of the thermalization of isolated orientational degrees of freedom 9] or, by using a directed beam of ultra-cold atoms, one can measure the scattering cross section, thus probing the dispersion interaction of nanoscale dielectrics [53].

Ground-state cooling of the nanoparticle would comprise a first step towards optomechanical experiments involving both the center-of-mass and the orientational degrees of freedom [54, 55]. Such deeply trapped particles can be used as point sources for orientation-dependent interference experiments [56, 57] by switching off rapidly the cavity [58, 59]. If the laser intensity is reduced adiabatically [60], on the other hand, a free quantum state of low kinetic and rotational energy can be generated. Finally, aligning many anisotropic particles in a single cavity mode might give rise to novel phenomena, such as a non-polar version of a gas of interacting dipoles [61, where synchronization of the dielectric's motion may be observable 22 .

\section{ACKNOWLEDGMENTS}

S. K. and M. A. acknowledge financial support from the Austrian Science Fund (FWF): P 27297 and W12103. We thank James Millen for stimulating discussions.
[1] O. M. Maragò, P. H. Jones, P. G. Gucciardi, G. Volpe, and A. C. Ferrari, Optical trapping and manipulation of nanostructures, Nat. Nanotechnol. 8, 807-819 (2013).

[2] O. Marago, P. Jones, F. Bonaccorso, V. Scardaci, P. Gucciardi, A. Rozhin, and A. Ferrari, Femtonewton force sensing with optically trapped nanotubes, Nano Lett. 8, 3211-3216 (2008).

[3] S. Olof, J. Grieve, D. Phillips, H. Rosenkranz, M. Yallop, M. Miles, A. Patil, S. Mann, and D. Carberry, Measuring nanoscale forces with living probes, Nano Lett. 12, 60186023 (2012).

[4] D. C. Moore, A. D. Rider, and G. Gratta, Search for Millicharged Particles Using Optically Levitated Microspheres, Phys. Rev. Lett. 113, 251801 (2014).

[5] G. Ranjit, M. Cunningham, K. Casey, and A. A. Geraci, Zeptonewton force sensing with nanospheres in an optical lattice, Phys. Rev. A 93, 053801 (2016).

[6] J. Gieseler, L. Novotny, and R. Quidant, Thermal nonlinearities in a nanomechanical oscillator, Nat. Phys. 9, 806-810 (2013).

[7] J. Millen, T. Deesuwan, P. Barker, and J. Anders, Nanoscale temperature measurements using non-equilibrium Brownian dynamics of a levitated nanosphere, Nat. Nano 9, 425429 (2014).

[8] J. Gieseler, R. Quidant, C. Dellago, and L. Novotny, Dynamic relaxation of a levitated nanoparticle from a nonequilibrium steady state, Nat. Nano 9, 358364 (2014).

[9] J. Millen and A. Xuereb, Perspective on quantum thermodynamics, New J. Phys. 18, 011002 (2016).

[10] A. Bassi, K. Lochan, S. Satin, T. Singh, and H. Ul- bricht, Models of wave-function collapse, underlying theories, and experimental tests, Rev. Mod. Phys. 85, 471527 (2013).

[11] M. Arndt and K. Hornberger, Testing the limits of quantum mechanical superpositions, Nat. Phys. 10, 271-277 (2014).

[12] G. Morigi, P. W. H. Pinkse, M. Kowalewski, and R. de Vivie-Riedle, Cavity Cooling of Internal Molecular Motion, Phys. Rev. Lett. 99, 073001 (2007).

[13] E. S. Shuman, J. F. Barry, and D. DeMille, Laser cooling of a diatomic molecule, Nature 467, 820-823 (2010).

[14] M. Yeo, M. T. Hummon, A. L. Collopy, B. Yan, B. Hemmerling, E. Chae, J. M. Doyle, and J. Ye, Rotational State Microwave Mixing for Laser Cooling of Complex Diatomic Molecules, Phys. Rev. Lett. 114, 223003 (2015).

[15] L. Paterson, M. MacDonald, J. Arlt, W. Sibbett, P. Bryant, and K. Dholakia, Controlled rotation of optically trapped microscopic particles, Science 292, 912-914 (2001).

[16] K. D. Bonin, B. Kourmanov, and T. G. Walker, Light torque nanocontrol, nanomotors and nanorockers, Opt. Express 10, 984-989 (2002).

[17] P. Jones, F. Palmisano, F. Bonaccorso, P. Gucciardi, G. Calogero, A. Ferrari, and O. Marago, Rotation detection in light-driven nanorotors, ACS Nano 3, 3077-3084 (2009).

[18] L. Tong, V. D. Miljkovic, P. Johansson, and M. Kall, Plasmon hybridization reveals the interaction between individual colloidal gold nanoparticles confined in an optical 
potential well, Nano Lett. 11, 4505-4508 (2010).

[19] M. Padgett and R. Bowman, Tweezers with a twist, Nat. Photonics 5, 343-348 (2011).

[20] Y. Arita, M. Mazilu, and K. Dholakia, Laser-induced rotation and cooling of a trapped microgyroscope in vacuum, Nat. Comm. 4, 2374 (2013).

[21] O. Brzobohatý, A. V. Arzola, M. Šiler, L. Chvátal, P. Jákl, S. Simpson, and P. Zemánek, Complex rotational dynamics of multiple spheroidal particles in a circularly polarized, dual beam trap, Opt. Express 23, 7273-7287 (2015).

[22] S. Simpson, L. Chvátal, and P. Zemánek, Synchronization of colloidal rotors through angular optical binding, Phys. Rev. A 93, 023842 (2016).

[23] S. E. S. Spesyvtseva and K. Dholakia, Trapping in a Material World, ACS Photonics 3, 719-736 (2016).

[24] S. Kuhn, P. Asenbaum, A. Kosloff, M. Sclafani, B. A. Stickler, S. Nimmrichter, K. Hornberger, O. Cheshnovsky, F. Patolsky, and M. Arndt, Cavity-Assisted Manipulation of Freely Rotating Silicon Nanorods in High Vacuum, Nano Lett. 15, 5604-5608 (2015).

[25] N. Kiesel, F. Blaser, U. Delić, D. Grass, R. Kaltenbaek, and M. Aspelmeyer, Cavity cooling of an optically levitated submicron particle, Proc. Nat. Acad. Sci. 110, 14180-14185 (2013).

[26] P. Asenbaum, S. Kuhn, S. Nimmrichter, U. Sezer, and M. Arndt, Cavity cooling of free silicon nanoparticles in high vacuum, Nat. Comm. 4 (2013).

[27] J. Millen, P. Fonseca, T. Mavrogordatos, T. Monteiro, and P. Barker, Cavity cooling a single charged levitated nanosphere, Phys. Rev. Lett. 114, 123602 (2015).

[28] O. Romero-Isart, M. L. Juan, R. Quidant, and J. I. Cirac, Toward quantum superposition of living organisms, New J. Phys. 12, 033015 (2010).

[29] D. Chang, K. Ni, O. Painter, and H. Kimble, Ultrahigh-Q mechanical oscillators through optical trapping, New J. Phys. 14, 045002 (2012).

[30] A. C. Pflanzer, O. Romero-Isart, and J. I. Cirac, Masterequation approach to optomechanics with arbitrary dielectrics, Phys. Rev. A 86, 013802 (2012).

[31] N. Voshchinnikov and V. Farafonov, Optical properties of spheroidal particles, Astrophysics and Space Science 204, 19-86 (1993).

[32] S. Simpson, Inhomogeneous and anisotropic particles in optical traps: Physical behaviour and applications, J. Quant. Spectrosc. Radiat. Transfer 146, 81-99 (2014).

[33] L. D. Cohen, R. D. Haracz, A. Cohen, and C. Acquista, Scattering of light from arbitrarily oriented finite cylinders, Appl. Opt. 22, 742-748 (1983).

[34] R. Schiffer and K. Thielheim, Light scattering by dielectric needles and disks, J. Appl. Phys. 50 (1979).

[35] H. van de Hulst, Light Scattering by Small Particles, Dover Publications - New York, 1981.

[36] T. Salzburger and H. Ritsch, Collective transverse cavity cooling of a dense molecular beam, New J. Phys. 11, 055025 (2009).

[37] J. D. Jackson, Classical Electrodynamics, Wiley, New York, 1999.

[38] S. M. Barnett and R. Loudon, On the electromagnetic force on a dielectric medium, J. Phys. B 39, S671 (2006).

[39] H. Stapelfeldt and T. Seideman, Colloquium, Rev. Mod. Phys. 75, 543-557 (2003).

[40] D. Pozar, Microwave Engineering, Wiley, 1997.

[41] D. Chang, C. Regal, S. Papp, D. Wilson, J. Ye,
O. Painter, H. Kimble, and P. Zoller, Cavity optomechanics using an optically levitated nanosphere, Proc. Nat. Acad. Sci. 107, 1005-1010 (2010).

[42] G. S. Agarwal, Quantum Optics, Cambridge University Press, 2013.

[43] A. R. Edmonds, Angular Momentum in Quantum Mechanics, Princeton University Press, Princeton, 1996.

[44] D. M. Brink and G. Satchler, Angular Momentum, Oxford Science Publications, 2002.

[45] G. S. Ezra, On the statistical mechanics of nonHamiltonian systems: the generalized Liouville equation, entropy, and time-dependent metrics, J. Math. Chem. 35, 29-53 (2004).

[46] G. Hechenblaikner, M. Gangl, P. Horak, and H. Ritsch, Cooling an atom in a weakly driven high-Q cavity, Phys. Rev. A 58, 3030-3042 (1998).

[47] H. Ritsch, P. Domokos, F. Brennecke, and T. Esslinger, Cold atoms in cavity-generated dynamical optical potentials, Rev. Mod. Phys. 85, 553-601 (2013).

[48] S. Habraken, W. Lechner, and P. Zoller, Resonances in dissipative optomechanics with nanoparticles: Sorting, speed rectification, and transverse cooling, Phys. Rev. A 87, 053808 (2013).

[49] M. C. Hohberger and K. Khaled, Cavity cooling of a microlever, Nature 432, 10021005 (2004).

[50] T. Li, S. Kheifets, and M. G. Raizen, Millikelvin cooling of an optically trapped microsphere in vacuum, Nat. Phys. 7, 527-530 (2011).

[51] J. Gieseler, B. Deutsch, R. Quidant, and L. Novotny, Subkelvin Parametric Feedback Cooling of a Laser-Trapped Nanoparticle, Phys. Rev. Lett. 109, 103603 (2012).

[52] J. Vovrosh, M. Rashid, D. Hempston, J. Bateman, and H. Ulbricht, Controlling the Motion of a Nanoparticle Trapped in Vacuum, arXiv preprint arXiv:1603.02917 (2016).

[53] T. Emig, N. Graham, R. L. Jaffe, and M. Kardar, Casimir Forces between Arbitrary Compact Objects, Phys. Rev. Lett. 99, 170403 (2007).

[54] M. Bhattacharya and P. Meystre, Using a LaguerreGaussian Beam to Trap and Cool the Rotational Motion of a Mirror, Phys. Rev. Lett. 99, 153603 (2007).

[55] H. Shi and M. Bhattacharya, Optomechanics based on angular momentum exchange between light and matter, arXiv preprint arXiv:1512.08989 (2015).

[56] B. W. Shore, P. Dömötör, E. Sadurní, G. Süssmann, and W. P. Schleich, Scattering of a particle with internal structure from a single slit, New J. Phys. 17, 013046 (2015).

[57] B. A. Stickler and K. Hornberger, Molecular rotations in matter-wave interferometry, Phys. Rev. A 92, 023619 (2015).

[58] O. Romero-Isart, A. C. Pflanzer, F. Blaser, R. Kaltenbaek, N. Kiesel, M. Aspelmeyer, and J. I. Cirac, Large Quantum Superpositions and Interference of Massive Nanometer-Sized Objects, Phys. Rev. Lett. 107, 020405 (2011).

[59] J. Bateman, S. Nimmrichter, K. Hornberger, and H. Ulbricht, Near-field interferometry of a free-falling nanoparticle from a point-like source, Nat. Commun. 5, 4788 (2014).

[60] P. Barker and M. Shneider, Cavity cooling of an optically trapped nanoparticle, Phys. Rev. A 81, 023826 (2010).

[61] S. A. Moses, J. P. Covey, M. T. Miecnikowski, B. Yan, B. Gadway, J. Ye, and D. S. Jin, Creation of a low- 
entropy quantum gas of polar molecules in an optical lattice, Science 350, 659-662 (2015). 